\title{
INFLUÊNCIA DO TAMANHO E DA FORMA DE FRAGMENTOS FLORESTAIS NA COMPOSIÇÃO DA TAXOCENOSE DE CRISOPÍDEOS (NEUROPTERA: CHRYSOPIDAE)
}

\author{
B. Souza1 ${ }^{1}$, R.I.F. Costa ${ }^{2}$, J.N.C. Louzada ${ }^{1}$
}

1Universidade Federal de Lavras, Departamento de Entomologia, CP 3037, CEP 37200-000, Lavras, MG, Brasil. E-mail: brgsouza@ufla.br

\section{RESUMO}

\begin{abstract}
Neste estudo objetivou-se verificar a composição da comunidade de crisopídeos (Neuroptera: Chrysopidae) em 15 fragmentos de floresta estacional semidecídua da região do Alto Rio Grande, sul do Estado de Minas Gerais, Brasil, assim como o efeito do tamanho e complexidade da forma desses fragmentos sobre a riqueza, abundância e diversidade das espécies. Os adultos de crisopídeos foram capturados com rede entomológica, durante uma hora, no interior dos fragmentos florestais até $50 \mathrm{~m}$ da borda. A área do fragmento foi obtida com auxílio de GPS, o qual gerou também a medida do perímetro, usados para cálculo da complexidade da forma. Foram coletados 154 espécimes de crisopídeos pertencentes a duas subfamílias, duas tribos, cinco gêneros e 17 espécies, com riqueza próxima de 23 espécies, estimada conforme a primeira aproximação de Jackknife. O gênero com maior diversidade de espécies foi Leucochrysa McLachlan, 1868 (Leucochrysini) (oito espécies); no entanto, Ceraeochrysa tucumana (Navás, 1919) (Chrysopini) foi a espécie mais abundante (45 exemplares). A riqueza de espécies não foi influenciada pela área ou complexidade da forma dos fragmentos.
\end{abstract}

PALAVRAS-CHAVE: Mata Atlântica, biodiversidade, Ceraeochrysa, Leucochrysa, predadores.

\section{ABSTRACT}

INFLUENCE OF SIZE AND FORM OF FOREST FRAGMENTS ON THE TAXOCENOSIS COMPOSITION OF GREEN LACEWINGS (NEUROPTERA: CHRYSOPIDAE). This study aimed to verify the lacewings taxocenosis (Neuroptera: Chrysopidae) in 15 semideciduous forest fragments in the Alto Rio Grande region, southern Minas Gerais State, Brazil, as well as the effect of the patches' size and complexity on the community structure. The adults were caught by sweep net, for one hour, inside the forest fragment distant $50 \mathrm{~m}$ from the edge. The area of the fragments was obtained using a GPS, which also generated the measure of the perimeter, used for calculation of the patch's complexity. A total of 154 lacewing specimens were collected belonging to 2 subfamilies, 2 tribes, 5 genera and 17 species. The estimated richness was 23 species, by the Jackknife1 method. Leucochrysa McLachlan, 1868 (Leucochrysini) was the genus with highest diversity (8 species). However, Ceraeochrysa tucumana (Navás, 1919) (Chrysopini) was the most abundant species ( 45 specimens). The species richness and abundance in the fragments studied was not influenced by area or complexity.

KEY WORDS: Atlantic Forest, biodiversity, Ceraeochrysa, Leucochrysa, predators.

\section{INTRODUÇÃO}

Desde o século XIX, a diversidade de insetos nos trópicos úmidos tem despertado o interesse de vários naturalistas como Bates, Wallace e Darwin. Mesmo após um século e meio, nossos conhecimentos acerca da dimensão da diversidade de insetos, bem como dos processos que a geram e a mantêm são rudimentares. Estima-se que a fração de insetos descritos nos trópicos seja inferior a 20\% (GODFRAY et al., 1999). O Brasil continua destacando-se na imprensa local e internacional devido aos altos níveis de devastação dos remanescentes de florestas tropicais, mesmo sabendo que a fragmentação desses ambientes ocasiona severos desequilíbrios ecológicos devido à redução da riqueza e diversidade de inúmeros táxons (Golden; CRIST, 1999; SAUNDERs et al., 1991).

\footnotetext{
${ }^{2}$ Fundação Educacional de Machado, Machado, MG, Brasil.
} 
Os insetos apresentam-se como um grupo importante na manutenção da estrutura dos fragmentos de florestas tropicais devido a sua elevada diversidade, ocupação de vários níveis tróficos e potencial de utilização no monitoramento das respostas de espécies às mudanças no habitat (KREMEN et al., 1993; Paoletti et al., 1999; Andersen et al., 2002; Progar; Schowalter, 2002). Embora alguns insetos apresentem rápida resposta às alterações no habitat, poucos estudos têm empregado esses organismos na avaliação de impactos ambientais (LAwTON et al., 1998; Novotny et al., 2002). Além da importância em estudos de biodiversidade e de impacto ambiental, a riqueza de insetos entomófagos em agroecossistemas é de fundamental importância para a estabilidade ecológica e a autoregulação, características dos ecossistemas naturais (Finke; Denno, 2004).

Tendo em vista que a maior parte dos remanescentes florestais encontra-se na forma de fragmentos e considerando a importância dos crisopídeos (Neuroptera: Chrysopidae) na regulação natural de populações de vários artrópodes fitófagos (SouzA; CARVALHO, 2002), este estudo teve como objetivo avaliar a riqueza de espécies e a estrutura da taxocenose desses predadores em fragmentos de floresta semidecídua na região do Alto Rio Grande, sul de Minas Gerais, assim como analisar a influência do tamanho e complexidade dos fragmentos sobre a riqueza e abundância de espécies.

\section{MATERIAL E MÉTODOS}

\section{Caracterização da área}

O estudo foi realizado no período de agosto a dezembro de 2002, em 15 fragmentos de floresta estacional semidecidual situados na região do Alto RioGrande, nos Municípios de BomSucesso e Lavras, sul de Minas Gerais. A escolha das áreas foi feita a partir de mapas (1:50.000) e ortofotos (1:10.000) da região, obtidos junto ao Instituto Estadual de Florestas - IEF e à Companhia Energética de Minas Gerais - CEMIG. Para a determinação da área e da forma dos fragmentos, percorreu-se o entorno de cada um deles, registrando, em um receptor GPS (Global Position System), as coordenadas geográficas geodésicas, definidas pela latitude e longitude. Os dados foram processados para a obtenção da área e do perímetro dos fragmentos. O perímetro foi utilizado no cálculo da complexidade da forma dos fragmentos.

Os fragmentos foram caracterizados pela fisionomia defloresta estacional semidecidual, inseridos no domínio da Mata Atlântica Sensu Lato (Tabela 1).

\section{Determinação da complexidade da forma dos fragmentos}

Para avaliar a complexidade da forma dos fragmentos, foi utilizado oíndice desenvolvido porPATTON (1975), através da seguinte fórmula:

$$
C=\frac{P}{\sqrt{\pi A}}
$$

em que: $C=$ índice de Patton para a complexidade de forma; $P=$ perímetro do fragmento $\mathrm{e}, A=$ área do fragmento.

Tabela 1 - Características dos fragmentos de floresta estacional semidecidual, conforme a área total, perímetro, complexidade e coordenadas geográficas. Região do Alto Rio Grande, sul de Minas Gerais. Agosto a dezembro de 2002.

\begin{tabular}{|c|c|c|c|c|c|}
\hline Fragmentos (amostras) & Área (ha) & Perímetro (m) & Complexidade* & Latitude (S) & Longitude $(\mathrm{W})$ \\
\hline 1 & 3,0 & 667 & 2,2 & $21^{\circ} 14^{\prime} 08^{\prime \prime}$ & $45^{\circ} 03^{\prime} 30^{\prime \prime}$ \\
\hline 2 & 5,8 & 1059 & 2,5 & $21^{\circ} 13^{\prime} 40^{\prime \prime}$ & $44^{\circ} 57^{\prime} 50^{\prime \prime}$ \\
\hline 3 & 6,1 & 1482 & 3,4 & $21^{\circ} 18^{\prime} 27^{\prime \prime}$ & $44^{\circ} 53^{\prime} 29^{\prime \prime}$ \\
\hline 4 & 7,5 & 1224 & 2,5 & $21^{\circ} 14^{\prime} 23^{\prime \prime}$ & $45^{\circ} 04^{\prime} 16^{\prime \prime}$ \\
\hline 5 & 8,0 & 1321 & 2,6 & $21^{\circ} 14^{\prime} 47^{\prime \prime}$ & $45^{\circ} 05^{\prime} 18^{\prime \prime}$ \\
\hline 6 & 10,0 & 1125 & 2,0 & $21^{\circ} 13^{\prime} 02^{\prime \prime}$ & $45^{\circ} 03^{\prime} 44^{\prime \prime}$ \\
\hline 7 & 11,0 & 2092 & 3,6 & $21^{\circ} 04^{\prime} 36^{\prime \prime}$ & $44^{\circ} 56^{\prime} 54^{\prime \prime}$ \\
\hline 8 & 12,1 & 1990 & 3,2 & $21^{\circ} 12^{\prime} 47^{\prime \prime}$ & $44^{\circ} 58^{\prime} 22^{\prime \prime}$ \\
\hline 9 & 13,0 & 1494 & 2,3 & $21^{\circ} 03^{\prime} 59^{\prime \prime}$ & $44^{\circ} 55^{\prime} 57^{\prime \prime}$ \\
\hline 10 & 13,3 & 1599 & 2,5 & $21^{\circ} 05^{\prime} 18^{\prime \prime}$ & $44^{\circ} 54^{\prime} 45^{\prime \prime}$ \\
\hline 11 & 14,2 & 1487 & 2,2 & $21^{\circ} 13^{\prime} 39^{\prime \prime}$ & $45^{\circ} 06^{\prime} 13^{\prime \prime}$ \\
\hline 12 & 15,7 & 2170 & 3,1 & $21^{\circ} 10^{\prime} 54^{\prime \prime}$ & $44^{\circ} 59^{\prime} 36^{\prime \prime}$ \\
\hline 13 & 16,1 & 1752 & 2,5 & $21^{\circ} 13^{\prime} 38^{\prime \prime}$ & $44^{\circ} 55^{\prime} 35^{\prime \prime}$ \\
\hline 14 & 16,6 & 1968 & 2,7 & $21^{\circ} 05^{\prime} 22^{\prime \prime}$ & $44^{\circ} 54^{\prime} 51^{\prime \prime}$ \\
\hline 15 & 17,6 & 2135 & 2,9 & $21^{\circ} 13^{\prime} 11^{\prime \prime}$ & $44^{\circ} 57^{\prime} 55^{\prime \prime}$ \\
\hline
\end{tabular}

*Valores obtidos pelo índice de PATton (1975). 
Quanto maior o valor do índice de Patton, maior a probabilidade de ocorrência de microambientes e maior a complexidade dos fragmentos; entretanto, maior será a proporção de biota em relação à área do fragmento, resultando num maior efeito de borda.

\section{Amostragem}

Em cada área amostral foi estabelecido um ponto inicial de coleta, situado no interior do fragmento a, aproximadamente, $50 \mathrm{~m}$ da borda. As coletas foram efetuadas semanalmente, sempre no período da tarde, com duração de uma hora, entre 5 de agosto e 9 de dezembro de 2002. Utilizou-se uma rede entomológica de $30 \mathrm{~cm}$ de diâmetro por $60 \mathrm{~cm}$ de comprimento confeccionada com tecidovoile. Os crisopídeos capturados foram colocados em tubos de acrílico e, no Laboratório de Recepção e Triagem de Material do Departamento de Entomologia da Universidade Federal de Lavras (UFLA), foram quantificados, montados em alfinete entomológico, etiquetados com os dados do local de coleta e identificados taxonomicamente.

\section{Análise dos dados}

O procedimento estatístico empregado na análise dos dados foi semelhante àquele utilizado porSANTOS (2004) para o estudo da estrutura da fauna de formigas em fragmentos florestais semelhantes.

\section{Caracterização da comunidade de Chrysopidae}

A fauna de crisopídeos foi caracterizada através da identificação das subfamílias, tribos, gêneros e espécies capturadas. A abundância dos crisopídeos foi calculada relacionando o número de exemplares de cada espécie com o número total de indivíduos capturados, enquanto a freqüência foi obtida pela ocorrência das espécies em cada amostra em relação ao número total de amostras.

Para o estudo da estrutura dessa comunidade nos fragmentos florestais utilizou-se a curva de rarefação de espécies, a partirdonúmeroabsolutodeespéciescoletadas em cada fragmento. Com o gráfico obtido a partir dessa curva, visualiza-seariqueza deespéciesobservada( Sobs).

A riqueza estimada de espécies foi obtida por meio da primeira aproximação de Jackknife, enquanto a diversidade de espécies foi obtida pelo índice de Shannon $\left(\mathrm{H}^{\prime}\right)$. Esses parâmetros foram obtidos através do programa EstimateS, versão 7.5, com 100 aleatorizações (CoLwell, 2005).

\section{Abordagem estatística}

No delineamento estatístico, os fragmentos foram considerados como repetições, sendo realizada ape- nas uma amostragem em cada um deles. Esse método tem como vantagem ofato de se ter homogeneidadeem relação à fisionomia vegetacional e, ao mesmo tempo, maior diversidade de hábitats, fornecendo amostras mais representativas do ponto de vista regional.

Para evidenciar a relação entre a área dos fragmentos e a complexidade da forma, utilizou-se o modelo de regressão linear com distribuição normal, descrita a partir da equação matemática: $y=a x+b$, em que: $y$ $=$ complexidade da forma dos fragmentos, $x=$ área dos fragmentos e $a$ e $b=$ coeficientes da regressão.

Na relação entre a área ou complexidade da forma dos fragmentos e a riqueza ou abundância de espécies utilizou-se, também, o modelo de regressão linear, sendo: $y=$ riqueza observada ou abundância das espécies, $x=$ área ou complexidade da forma dos fragmentos e $a$ e $b=$ coeficientes da regressão.

\section{RESULTADOS E DISCUSSÃO}

\section{Composição e diversidade da taxocenose de crisopídeos}

Foram coletados 154 espécimes de crisopídeos pertencentes a duas subfamílias, cinco gêneros e 17 espécies (Tabela 2). A subfamília Notochrysinae foi representada pelo gêneroAsthenochrysaeChrysopinae por Ceraeochrysa, Chrysopodes e Chrysoperla (Chrysopini) eLeucochrysa (Leucochrysini). Dentreos Chrysopinae, o gênero Leucochrysa foi o mais diverso, com oito espécies $(47,1 \%$ do total de espécies capturadas) e freqüência de $93,3 \%$. O segundo gênero mais diverso foi Ceraeochrysa, com representantes de quatro espécies, seguido por Chrysopodes (três espécies) e Chrysoperla, representado por C. externa. Por outro lado, quando analisada a abundância das tribos, Chrysopini foi a mais abundante com $72,1 \%$ do total de espécimes coletados e freqüência de 100,0\%.

Embora Chrysopini seja considerada a tribo queconcentraa maior diversidadedetáxons registradosmundialmente (BROOKS; BARNARD, 1990), a riqueza de espécies verificada neste trabalho foi semelhante em ambas as tribos: Chrysopini e Leucochrysini. Trata-se de uma constataçãoesperada, dadoqueLeucochrysiniconstitui uma tribo com ampla ocorrência na região Neotropical e apresenta alta diversidade de espécies, sobretudo do gênero Leucochrysa. De acordo com ADAMs; PENNY (1986), para a região Amazônica é citada a ocorrência de 141 espécies desse gênero, distribuídas nos subgêneros Leucochrysa eNodita com36e105espécies, respectivamente. Nessa região, a diversidade de Leucochrysini pode aproximar-se ou até mesmo superar a diversidade de Chrysopini. Atéomomento, estãoregistradas 73 espécies de Leucochrysa para o Brasil, incluindo 25 espécies recentemente descritas por FreITAS; PENNY (2001). 
Tabela 2 - Abundância e freqüência dos táxons (Neuroptera: Chrysopidae) coletados em fragmentos de floresta estacional semidecidual, na região do Alto Rio Grande, sul de Minas Gerais. Agosto a dezembro de 2002.

\begin{tabular}{|c|c|c|c|c|}
\hline \multirow[t]{2}{*}{ Táxons } & \multicolumn{2}{|c|}{ Abundância } & \multicolumn{2}{|c|}{ Freqüência } \\
\hline & № & $(\%)$ & № & $(\%)$ \\
\hline Subfamília Nothochrysinae Navás, 1910 & 1 & 0,7 & 1 & 6,7 \\
\hline Gênero Asthenochrysa Adams e Penny, 1992 & 1 & 0,7 & 1 & 6,7 \\
\hline A. viridula (Adams, 1978) & 1 & 0,7 & 1 & 6,7 \\
\hline Subfamília Chrysopinae Schneider, 1851 & 153 & 99,4 & 15 & 100 \\
\hline Tribo Chrysopini Schneider, 1851 & 111 & 72,1 & 15 & 100 \\
\hline Gênero Ceraeochrysa Adams, 1982 & 72 & 46,8 & 13 & 86,7 \\
\hline Ceraeochrysa sp.1 & 19 & 12,3 & 6 & 40,0 \\
\hline Ceraeochrysa sp.2 & 1 & 0,7 & 1 & 6,7 \\
\hline C. tenuicornis Adams \& Penny, 1987 & 7 & 4,6 & 3 & 20,0 \\
\hline C. tucumana (Navás, 1919) & 45 & 29,2 & 14 & 93,3 \\
\hline Gênero Chrysoperla Steinmann, 1964 & 5 & 3,3 & 2 & 13,3 \\
\hline C. externa (Hagen, 1861) & 5 & 3,3 & 2 & 13,3 \\
\hline Gênero Chrysopodes Navás, 1913 & 34 & 22,1 & 8 & 53,3 \\
\hline Subgênero Chrysopodes Navás, 1913 & 30 & 19,5 & 8 & 53,3 \\
\hline Chrysopodes (C.)sp.1 & 29 & 18,8 & 9 & 60,0 \\
\hline Chrysopodes (C.)sp.2 & 1 & 0,7 & 1 & 6,7 \\
\hline Subgênero Neosuarius Adams \& Penny, 1987 & 4 & 2,7 & 2 & 13,3 \\
\hline Chrysopodes divisa (Walker, 1853) & 4 & 2,6 & 2 & 13,3 \\
\hline Tribo Leucochrysini Adams, 1978 & 42 & 27,3 & 14 & 93,3 \\
\hline Gênero Leucochrysa McLachlan , 1868 & 42 & 27,3 & 14 & 93,3 \\
\hline Subgênero Nodita Navás, 1916 & 38 & 24,7 & 13 & 86,7 \\
\hline Leucochrysa (N.) sp.1 & 21 & 13,6 & 10 & 66,7 \\
\hline Leucochrysa (N.) sp.2 & 5 & 3,3 & 2 & 13,3 \\
\hline Leucochrysa (N.) sp.3 & 2 & 1,3 & 1 & 6,7 \\
\hline Leucochrysa (N.) sp.4 & 5 & 3,3 & 4 & 26,7 \\
\hline Leucochrysa (N.) sp.5 & 5 & 3,3 & 3 & 20,0 \\
\hline Subgênero Leucochrysa MacLachlan, 1868 & 4 & 2,6 & 3 & 20,0 \\
\hline Leucochrysa magnifica (Banks, 1920) & 1 & 0,7 & 1 & 6,7 \\
\hline Leucochrysa varia (Schneider, 1851) & 1 & 0,7 & 1 & 6,7 \\
\hline Leucochrysa (L.) sp.1 & 2 & 1,3 & 1 & 6,7 \\
\hline Total & 154 & 100,0 & 15 & 100,0 \\
\hline
\end{tabular}

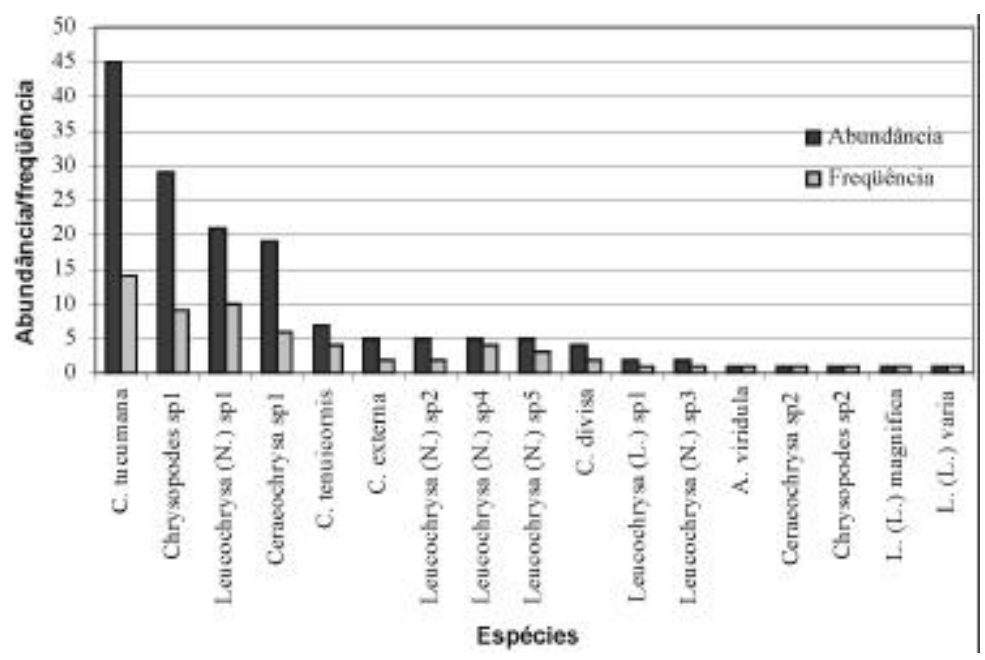

Fig. 1 - Abundância e freqüência das espécies decrisopídeos (Neuroptera: Chrysopidae) coletadas em fragmentos de floresta estacional semidecidual, na região do Alto Rio Grande, sul de Minas Gerais. Agosto a dezembro de 2002. 
Com relação a Nothochrysinae, foi coletado apenas um espécime macho do gênero Asthenochrysa (0,7\% do total de indivíduos capturados). Trata-se de uma espécie rara, descrita por ADAms (1978) comoHypochrysa viridula, a qual foi posteriormente transferida para o novo gênero Asthenochrysa Adams \& Penny, 1992, o que levou à nova combinaçãoAsthenochrysa viridula(Adams, 1978). No Brasil, há registros de ocorrência dessa espécie para Linhares, ES, e Serra do Caraça, MG (Adams; Penny, 1992).

A espécie mais abundante nos fragmentos florestais foi Ceraeochrysa tucumana (Navás, 1919), com 45 exemplares, seguida por Chrysopodes (C.) sp.1, Leucochrysa (N.) sp.1 e Ceraeochrysa sp.1, com 29, 21 e 19 exemplares, respectivamente. Por outro lado, muitas espécies amostradas apresentaram baixa abundância, cinco delas representadas por apenas um indivíduo e duas espécies com apenas dois exemplares (Fig. 1).

Apesar de utilizar literatura pertinente para a identificação das espécies de Chrysopidae da região Neotropical (AdAms; PennY, 1987; Freitas; PenNy, 2001) e contar com a orientação de especialistas na taxonomia do grupo, não foi possível a identificação específica de alguns táxons, podendo tratar-se de espécies novas (Tabela 2). Estudos serão conduzidos no sentido de efetuar a confirmação dos novos táxons e, se for o caso, sua posterior descrição. Os casos mais complexos referem-se aos táxons do gênero Leucochrysa, cujas descrições originais são sumárias e, em muitos casos, baseadas apenas em caracteres morfológicos externos, sem descrição das estruturas da genitália, o que justifica a realização de revisão do gênero.

\section{Indicadores de biodiversidade}

A curva de rarefação de espécies, construída a partir da riqueza observada (Sobs), sugere que as 15 amostragens não foram suficientes para a realização de um inventário preciso das espécies existentes nos fragmentos. De acordo com Colwell; Coddington (1994), quando essa curva é caracterizada por uma reta ascendente com tendência para um número infinito de espécies, ela indica que as condições ideais de esforço amostral ainda não foram obtidas. A curva ideal deverá ter uma distribuição assintótica, predizendo que, em uma determinada condição (ex. tempo decoleta), o esforço amostral para coletar " $n$ " espécies foi obtido. Desse modo, a tendência ascendente verificada na curva da riqueza observada (Fig. 2) indica a necessidade de um maior esforço amostral para a obtenção de um inventário capaz de fornecer o valor mais próximo possível do número real de espécies " $N$ " existentes nos fragmentos.
A estimativa da riqueza pelo índice de Jackknife1 foi de 22,6 espécies, indicando que, em média, poderiam ser observadas outras 5,6 espécies de crisopídeos além das 17 coletadas nos levantamentos efetuados (Fig. 2).

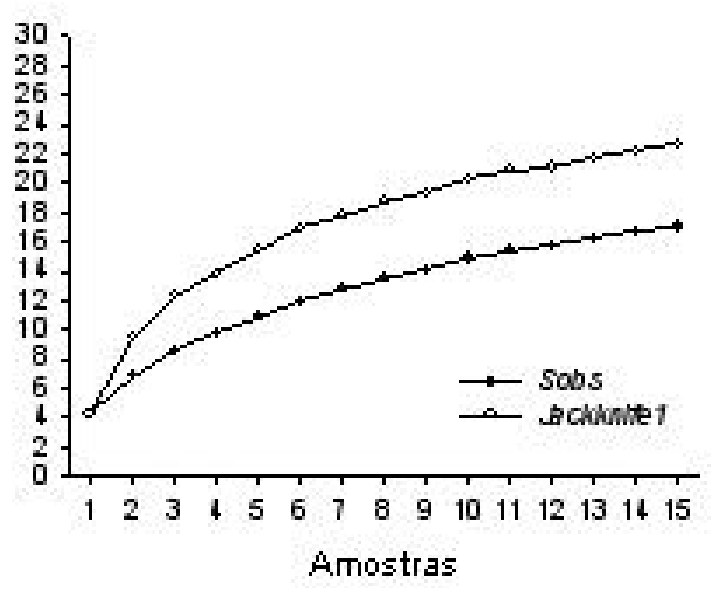

Fig. 2 - Riqueza observada (Sobs) e estimada (Jackknife1)de espécies de crisopídeos (Neuroptera: Chrysopidae) coletadas em fragmentos de floresta estacional semidecidual, na região do Alto Rio Grande, sul de Minas Gerais. Agosto a dezembro de 2002.

Para os fragmentos estudados, observaram-se valores do índice de diversidade de Shannon $\left(\mathrm{H}^{\prime}\right)$ entre 0,41 e 1,88 (Tabela 3). Analisando esses valores de modo isolado, pode-se considerar que a diversidade da comunidade de crisopídeos nos fragmentos florestais, nas condições em que se realizou este estudo, foi baixa, uma vez que os valores obtidos pelo índice de Shannon podem variar entre zero e cinco (KREBS, 1998), sendo o limite inferior atribuído aos ambientes com ausência de diversidade e o limite superior àqueles como a máxima diversidade obtida em condições naturais.

Por outro lado, se ao invés de analisar a diversidade em cada local separadamente, esse parâmetro for considerado no conjunto dos 15 fragmentos estudados, serão obtidos os números totais de 17 espécies e 154 indivíduos, com uma estimativa de diversidade $\mathrm{H}^{\prime}$ equivalente a 4,0 (Tabela 3).

GOLDEN; CRIST (1999) avaliaram os efeitos da fragmentação do habitat na comunidade de insetos associados ao dossel das plantas, nos meses de julho e setembro e constataram que, em julho, a riqueza de espécies nos fragmentos foi inferior à encontrada nos habitats não fragmentados e, em setembro, similares; não houve diferença com relação à abundância entre ambos os meses. Concluiu-se que a menor riqueza observada emjulho foi devida à ausência das espécies raras, as quais não impactaram os resultados da abundância. 
Tabela 3 - Número, abundância eíndice de diversidade de Shannon $\left(\mathrm{H}^{\prime}\right)$ de espécies de crisopídeos (Neuroptera: Chrysopidae) coletadas em fragmentos de floresta estacional semidecidual, na região do Alto Rio Grande, sul de Minas Gerais. Agosto a dezembro de 2002.

\begin{tabular}{|c|c|c|c|}
\hline $\begin{array}{c}\text { Fragmentos } \\
\text { (Amostras) }\end{array}$ & $\begin{array}{c}\mathrm{N}^{\mathrm{o}} \text { de espécies } \\
\text { observadas }\end{array}$ & Abundância & $\begin{array}{c}\text { Índice de } \\
\text { Shannon }\left(\mathrm{H}^{\prime}\right)\end{array}$ \\
\hline 1 & 2 & 5 & 0,50 \\
\hline 2 & 2 & 7 & 0,41 \\
\hline 3 & 3 & 14 & 1,06 \\
\hline 4 & 7 & 15 & 1,88 \\
\hline 5 & 5 & 11 & 1,52 \\
\hline 6 & 3 & 4 & 1,04 \\
\hline 7 & 3 & 6 & 1,01 \\
\hline 8 & 4 & 21 & 1,18 \\
\hline 9 & 7 & 12 & 1,86 \\
\hline 10 & 5 & 12 & 1,52 \\
\hline 11 & 4 & 10 & 1,37 \\
\hline 12 & 3 & 11 & 0,99 \\
\hline 13 & 5 & 9 & 1,52 \\
\hline 14 & 4 & 8 & 1,32 \\
\hline 15 & 4 & 9 & 1,27 \\
\hline Total & 17 & 154 & \\
\hline
\end{tabular}

\section{Influência do tamanho e complexidade dos frag- mentos sobre a riqueza de espécies}

As áreas de fragmentos de floresta variaram entre 3,0 e 17,6 ha e o índice de complexidade apresentou valores entre 2,0 e 3,6 (Tabela 1). Não foi constatada relação significativa entre a área e a complexidade da forma nos fragmentos estudados. Esses resultados diferem daqueles obtidos porSANTOS (2004), que verificou uma correlação positiva entre o aumento na área e a complexidade da forma de fragmentos com fitofisionomias semelhantes.

Otamanho do fragmento não teve influência sobre a riqueza e diversidade dos crisopídeos. Em estudos semelhantes conduzidos porSANTOS (2004) com espécies de Formicidae (Hymenoptera) e por LouzADA (2000) eSCHIFfLer(2003) com espécies deScarabaeidae (Coleoptera), também não foi constatada correlação entre a riqueza e a área dos fragmentos florestais estudados.

Quanto à complexidade da forma dos fragmentos, também não foi observada correlação significativa com a riqueza ou abundância decrisopídeos.SCHIFFLER (2003) não constatou correlação entre a riqueza de escarabeídeos e complexidade de fragmentos florestais na região do Alto Rio Grande, $\mathrm{MG}$, enquanto que LOUZADA (2000) observou correlação positiva entre a riqueza de escarabeídeos e a complexidade da forma em fragmentos florestais no Município de Viçosa,
MG, e SANTOS (2004) observou correlação negativa entre o aumento da complexidade e o número de espécies de formigas presentes em fragmentos florestais no sul de Minas Gerais.

Esses resultados corroboram o estudo conduzido por DidHAm etal. (1998), no qual coleópteros da AmazôniaCentralpertencentesadiferentesgrupostaxonômicos e/ou tróficos apresentaram respostas distintas à fragmentação do habitat. Desse modo, pode-se inferir que a correlaçãoentrea complexidade da forma ea riqueza de espécies pode ser influenciada pelas respostas de diferentes táxons à mesma fisionomia vegetacional, ou até mesmodegrupos taxonômicossemelhantesem diferentes sítios amostrais.

Os resultados obtidos nesta pesquisa indicam que a riqueza e a abundância dos crisopídeos não estão diretamente correlacionadas à extensão ou complexidade do fragmento, podendo ser influenciadas por outros fatores que atuam localmente, como a heterogeneidade do habitat, a disponibilidade de alimento e o microclima no interior dos fragmentos. A importância dos microhabitats para os neurópteros foi evidenciada por STELz; DevetaK (1999) ao afirmarem que, em algumas espécies desse grupo, o desenvolvimento larval pode ser restrito a um pequeno número, ou até mesmo a uma única espécie vegetal.

\section{CONCLUSÕES}

1. A riqueza e a diversidade dos crisopídeos não foram influenciadas pelo tamanho dos fragmentos florestais e revelaram que os fragmentos com menor área também são relevantes do ponto de vista da conservação desses artropódes.

2. A complexidade da forma dos fragmentos não influenciou a riqueza e abundância de crisopídeos, o que evidencia o efeito de fatores locais sobre a diversidade desses inimigos naturais.

\section{AGRADECIMENTOS}

Ao Conselho Nacional de Desenvolvimento Científico e Tecnológico - CNPq, pela concessão de bolsa de estudo ao segundo autor e à Fundação de Apoio ao Ensino e Pesquisa de Minas Gerais - FAPEMIG, pela concessão de recursos empregados na condução do estudo.

\section{REFERÊNCIAS}

ADAMS, P.A. A new species of Hypochrysa and a new subgenus and species of Mallada (Neuroptera:

Chrysopidae). The Pan-Pacific Entomologist, v.54, n.4, p.292-296, 1978. 
ADAMS, P.A.; PENNY, N.D. Faunal relations of Amazonian Chrysopidae. In: GEEP J.; ASPÖCK, H.; HÖLZEL, H. (Ed.). Recent research in neuropterology: proceedindgs of the second international symposium on neuropterology. Graz, 1986, p.119-124.

ADAMS, P.A.; PENNY, N.D. Neuroptera of the Amazon Basin II. Introducion and Chrysopini. Acta Amazonica, v.15, n.3/4, p.413-479, 1987.

ADAMS, P.A.; PENNY, N.D. A new genera of Nothochrysinae from south América (Neuroptera: Chrysopidae). The Pan-Pacific Entomologist, v.68, n.3, p.216-221, 1992.

ANDERSEN, A.N.; HOFFMANN, B.D.; MÜLLER, W.J.; GRIFFITHS, A.D. Using ants as bioindicators in land management: simplifying assessment of ant community response. Journal of Applied Ecology, v.39, n.1, p.8-17, 2002.

BROOKS, S.J.; BARNARD, P.C. The green lacewings of the word: a generic review (Neuroptera: Chrysopidae). Bulletin of the British Museum Natural History, v.59, n.2, p.117-286, 1990 .

COLWELL, R.K. EstimateS 7. 5 user's guide. Storrs: University of Connecticut. Disponível em: <http:// viceroy. eeb. uconn.edu/estimates>. Acesso em: 14 ago. 2005.

COLWELL, R.K.; CODDINGTON, J.A. Estimating terrestrial biodiversity through extrapolation. Philosophical Transactions of the Royal Entomological Society of London, v.345, n.1311, p.101-118, 1994.

DIDHAM, R.K.; LAWTON, J.H.; HAMMOND, P.M.; EGGLETON, P. Trophic structure stability and extinction dynamics of beetles (Coleoptera) in tropical forest fragments. Philosophical Transactions of the Royal Entomological Society of London, v.353, n.1367, p.437-451, 1998.

FINKE, D.L.; DENNO, R.F. Predator diversity dampens trophic cascades. Nature, v.429, n.6990, p.407-410, 2004.

FREITAS, S. DE; PENNY, N.D. The green lacewings (Neuroptera: Chrysopidae) of brazilian agroecossystems. Proceedings of the California Academy of Sciences, v.52, n.19, p.245-395, 2001.

GODFRAY, H.C.; LEWIS, O.T.; MEMMOT, J. Studying insect diversity in the tropics. Philosophical Transactions of the Royal Entomological Society of London, v.354, n.1391, p.1881-1824, 1999.

GOLDEN, D.M.; CRIST, T.O. Experimental effects of fragmentation on old field canopy insects: community, guild and species responses. Oecologia, v.118, n.3, p.371380, 1999.
KREBS, C.J. Ecological methodology. New York: AddisonWelsey Educational Publishers, 1998. 581p.

KREMEN, C.; COLWELL, R.K.; ERWIN, T.L.; MURPHY, D.D.; NOSS, R.F.; SAJAYAN, M.A. Terrestrial arthropod assemblages: their use in conservation planning. Conservation Biology, v.7, n.4, p.796-808, 1993.

LAWTON, J.H.; BIGNELL, D.E.; BOLTON, B.; BLOEMERS, G.F.; EGGLETON, P.; HAMMOND, P.M.; HODDA, M.; HOLT, R.D.; LARSEN, T.B.; MAWDSLEY, N.A.; STORK, N.E.; SRIVASTAVA, D.S.; WATT, A.D. Biodiversity inventories, indicator taxons and effects of hábitat modification in tropical forest. Nature, v.391, n.6662, p.72-76, 1998.

LOUZADA, J.N.C. Efeitos da fragmentação florestal sobre a comunidade de Scarabaeidae (Insecta: Coleoptera). 2000. 95p. Tese (Doutorado em Entomologia) - Universidade Federal de Viçosa, Viçosa, 2000.

NOVOTNY, V.; BASSET, Y.; MILLER, S.E.; WEIBLEN, G.D.; BREMER, B.; CIZEK, L.; DROZD, P. Low host specificity of herbivorous insects in a tropical forest. Nature, v.416, n.6883, p.841-844, 2002.

PAOLETTI, M.G.; DUNXIAO, H.; MARC, P.; NINGXING, H.; WENLIANG, W. Arthropods as bioindicators in agroecossystems of jiang Han Plain, Qianjiang City Hubei China. Critical Reviews in Plant Sciences, v.18, n.3, p.457-465, 1999.

PATTON, D.R. A diversity index for quantifying hábitat edge. Wildlife Society Bulletin, v.3, p.171-173, 1975.

PROGAR, R.A.; SCHOWALTER, T.D. Canopy arthropod assemblages along a precipitation and latitudinal gradient among Douglas-fir Pseudotsuga menziesii forests in the Pacific Northwest of the United States. Ecography, v.25, n.2, p.129-138, 2002.

SANTOS, M. S. Estrutura das comunidades de formigas (Hymenoptera: Formicidae) em fragmentos de floresta semidecídua. 2004. 97p. Dissertação (Mestrado em Agronomia/Entomologia) - Universidade Federal de Lavras, Lavras, 2004.

SAUNDERS, D.A.; HOBBS, R.J.; MARGULES, C.R. Biological consequences of ecosystem fragmentation: a review. Conservation Biology, v.5, n.1, p.18-32, 1991.

SCHIFFLER, G. Fatores determinantes da riqueza local de espécies de Scarabaeidae (Insecta: Coleoptera) em fragmentos de floresta estacional semidicídua. 2003. 68p. Dissertação (Mestrado em Agronomia/ Entomologia) - Universidade Federal de Lavras, Lavras, 2003. 
SOUZA, B.; CARVALHO, C.F. Population dynamics and seasonal occurrence of adults of Chrysoperla externa (Hagen, 1861) (Neuroptera: Chrysopidae) in a citrus orchard in southern Brazil. Acta Zoologica Academiae Scientiarum Hungaricae, v.48, p.301-310, 2002. Suplemento 2 .
STELZL, M.; DEVETAK, D. Neuroptera in agricultural ecosystems. Agriculture, Ecossistems and Environment, v.74, n.1/3, p.305-321, 1999.

Recebido em 10/4/07

Aceito em 18/8/08 